\title{
The expression of multiple cancer/testis antigens can potentially be used to detect circulating disease and clonal evolution in the peripheral blood of multiple myeloma patients
}

\author{
Karen Shires ${ }^{1}$, Teagan Van Wyk ${ }^{2}$, Kirsty Wienand ${ }^{3}$ \\ ${ }^{1}$ Division of Haematology, Department of Pathology, University of Cape Town and National Health Laboratory Service/Groote \\ Schuur Hospital, ${ }^{2}$ Department of Medicine, University of Cape Town, Cape Town, South Africa, ${ }^{3}$ Division of Medical Oncology, \\ Dana-Farber Cancer Institute, Boston, MA, USA
}

p-ISSN 2287-979X / e-ISSN 2288-0011 https://doi.org/10.5045/br.2021.2020335 Blood Res 2021;56:156-165.

Received on December 29, 2020

Revised on June 16, 2021

Accepted on July 8, 2021

\author{
*This study was supported by a grant from \\ National Health Laboratory Research \\ Trust, AstraZeneca Research Trust and \\ South African Medical Research Council. \\ Correspondence to \\ Karen Shires, Ph.D. \\ Division of Haematology, UCT Medical \\ School, Anzio Road, Observatory 7221, \\ Cape Town, South Africa \\ E-mail: Karen.shires@uct.ac.za \\ (C) 2021 Korean Society of Hematology
}

\begin{abstract}
Background
It is thought that cancer/testis antigens (CTAs) are expressed in a cascade-like manner in multiple myeloma as the disease progresses. In this pilot study, we investigated the co-expression of several CTAs in the peripheral blood (PB) during patient therapy to establish whether monitoring multiple CTAs allows for the prediction of relapse and clonal evolution.
\end{abstract}

\begin{abstract}
Methods
We examined the co-expression of $M A G E C 1, M A G E A 3, P R A M E$, and $B A G E 2$ via quantitative reverse transcription-polymerase chain reaction (qRT-PCR) duplex assays in the PB mononuclear cells of 10 patients on chemotherapy at 3-month intervals, and correlated the levels to those of two basic clinical monitoring markers, serum $\beta$-2-microglobulin and serum $M$ protein. Clonal evolution was investigated using flow cytometry to label the circulating malignant stem cell components with MAGEC1, PRAME, and MAGEA3 antibodies.
\end{abstract}

\section{Results}

Simultaneous monitoring of $M A G E C 1 / P R A M E$ provided sensitive detection of circulating malignant cells in easily accessible PB samples; transcript levels increased prior to changes in indicators of clinical relapse. While MAGEA3/BAGE2 expression levels did not offer earlier prediction of relapse, they provided insight into significant changes occurring within the malignant cell population; the addition of either CTA to a MAGEC1-monitoring panel allowed for better classification of the relapse event (clonal evolution), which in turn could potentially guide treatment strategies in the future.

\section{Conclusion}

This pilot study supports the novel idea of determining the levels and CTA expression patterns of the total circulating malignant cell population (pro-B/pre-B stem cell progenitors and proliferating plasma cells) as an alternate disease monitoring methodology.

Key Words CTA, MAGEC1, Myeloma, Cascade, Monitoring, PRAME

\section{INTRODUCTION}

Multiple myeloma (MM) is characterized by the accumulation of abnormal clonal plasma cells (PCs) in the bone marrow (BM), which results in BM failure. Treatment includes chemotherapeutic drugs and autologous stem cell transplantation (ASCT), and due to significant advancements in the last decade, most patients achieve a complete response (CR) and high overall survival (OS) rates. However, MM remains an incurable disease, and most patients relapse within five years [1, 2]. Standard monitoring assays such as serum and urine paraprotein (M-protein) detection in the peripheral blood (PB) and the percentage of PCs in the BM are used to define $\mathrm{CR}[3,4]$. However, these methods are limited in their sensitivity $[5,6]$ and newer methods have been 
developed to detect and characterize abnormal PCs including multiparametric flow cytometry analysis (EUROFLOW) (MFC) and polymerase chain reaction (PCR)/next generation sequencing (ASO-PCR and NGS) to detect clonal B cell receptor rearrangements of specific abnormal PC clones [7]. These methods have detection limits of $0.01-0.001 \%$ of PC populations and have indicated that minimal residual disease (MRD) is present (defined as abnormal PCs) in the BM of patients with $\mathrm{CR}$, and that this MRD is responsible for clinical relapses [1, 6, 8-11].

These newer methods have their limitations, as they require patient-specific adaptations and BM aspirate samples; additionally, they only assess the PC burden [7], and thus do not reflect the levels of the total malignant cell population that includes a stem cell component [12-16]. Circulating malignant cells lead to extramedullary disease; thus, monitoring their levels and gene expression profiles may provide a novel tool for assessing relapse and disease progression risk during therapy. A simplified quantitative reverse-transcription PCR (qRT-PCR) approach using PB samples that is applicable in all $\mathrm{MM}$ patients may help overcome the limitations of the current methods.

$\mathrm{MM}$ is a heterogeneous disease and a common molecular signature has not been established yet; however, we and others have shown a distinct relationship between the num- ber and type of cancer/testis antigens (CTAs) expressed with advancing disease, and suggested a cascade-like expression pattern [17-20]. Additionally, the CTA "melanoma associated antigen C1" (MAGEC1) was identified as a diagnostic marker of symptomatic MM (SMM); it was the most frequently expressed CTA in MM, and was found to be expressed in both early and advanced disease [20, 21]. A pro-B/pre-B stem cell progenitor has been reported to express this protein, implicating $M A G E C 1$ in carcinogenesis; thus, these early stem cells circulating in the $\mathrm{PB}$ may be the malignant progenitor cells that function as the feeder population in MM, in addition to proliferating PC populations that are detected in some patients [15]. MACEC1 expression reflects treatment responses and changes in serum $\mathrm{M}$ protein and serum beta-2-microglobulin $\left(\mathrm{S} \beta_{2} \mathrm{M}\right)$ levels. Thus, qRT-PCR or flow cytometry monitoring of MACEC1 is relevant in chemotherapy patients, and significantly high sensitivity to low levels of disease was observed with this approach [21].

Our previous studies demonstrated that the CTA "preferentially expressed antigen of melanoma" ( $P R A M E)$ was expressed in early stage disease (from stage 1), $M A G E A 3$ in advanced disease (stages 2/3), and BAGE2 exclusively in stage 3 disease [20]. Whether the expression of these genes occurs in different clones or represents clonal evolution has not yet been established. Therefore, we wanted to investigate

Table 1. Diagnostic characteristics of multiple myeloma (MM) patients (modified from Shires and Wienand, 2016 [21]).

\begin{tabular}{|c|c|c|c|c|c|c|c|c|c|}
\hline \multirow{2}{*}{$\begin{array}{l}\text { Patient } \\
\text { No. }\end{array}$} & \multirow{2}{*}{$\begin{array}{l}\text { Age, } \\
\text { sex }\end{array}$} & \multirow{2}{*}{$\begin{array}{l}\text { Disease } \\
\text { subtype } \\
(\lg \kappa / \lg \lambda)^{a)}\end{array}$} & \multirow{2}{*}{$\begin{array}{l}\text { Disease stage } \\
\text { at diagnosis } \\
\text { CRAB } \\
\text { features }^{\mathrm{c})}\end{array}$} & \multirow{2}{*}{ Treatment } & \multirow{2}{*}{$\begin{array}{l}\text { Sampling } \\
\text { period } \\
\text { (mo) }\end{array}$} & \multicolumn{4}{|c|}{$\%$ MAGEC1 cells at diagnosis in PB } \\
\hline & & & & & & Total $^{\mathrm{e})}$ & CD34+ & CD19+ & $\mathrm{CD} 138+{ }^{f}$ \\
\hline 1 & $66, \mathrm{~F}$ & $\lg G \lambda$ & $2, \mathrm{RAB}$ & $\begin{array}{l}\text { Cyclophosphamide+dexamethasone, } \\
\text { localized radiation }\end{array}$ & $12^{\text {d) }}$ & 1.29 & 0.71 & 0.46 & 0.12 \\
\hline 2 & $80, M$ & $\lg A \lambda$ & 3, CRAB & $\begin{array}{l}\text { Alternating between } \\
\text { cyclophosphamide+dexamethasone } \\
\text { and melphalan+prednisone }\end{array}$ & $9^{\text {d) }}$ & 1.35 & 0.43 & 0.52 & 0.41 \\
\hline 3 & $73, \mathrm{~F}$ & $\lg G \lambda$ & $1, \mathrm{AB}$ & $\begin{array}{l}\text { Cyclophosphamide for one month, } \\
\text { thereafter no myeloma treatment }\end{array}$ & 24 & 1.82 & 0.86 & 0.86 & 0.22 \\
\hline 4 & $73, \mathrm{~F}$ & $\operatorname{lgG\kappa }$ & $1, B$ & Melphalan + prednisone & 24 & 1.99 & 1.00 & 0.93 & 0.06 \\
\hline 6 & $33, \mathrm{M}$ & $\operatorname{lgG\kappa }$ & $2, \mathrm{AB}$ & $\begin{array}{l}\text { Alternating between } \\
\text { cyclophosphamide+dexamethasone } \\
\text { and melphalan+ prednisone }\end{array}$ & $18^{\mathrm{d})}$ & 1.15 & 0.59 & 0.43 & 0.13 \\
\hline 7 & $66, M$ & $\operatorname{lgG\kappa }$ & $2, A B$ & $\begin{array}{l}\text { Alternating between } \\
\text { cyclophosphamide+prednisone } \\
\text { and melphalan+ prednisone, } \\
\text { localized radiation }\end{array}$ & 24 & 1.24 & 0.41 & 0.45 & 0.29 \\
\hline 8 & $49, \mathrm{~F}$ & $\operatorname{IgA\kappa }$ & 3, RAB & $\begin{array}{l}\text { Cyclophosphamide + dexamethasone } \\
\text { as well as localized radiation }\end{array}$ & $12^{\mathrm{d})}$ & 1.46 & 0.42 & 0.75 & 0.26 \\
\hline 9 & $83, F$ & $\operatorname{lgG\kappa }$ & $1, A B$ & $\begin{array}{l}\text { Cyclophosphamide+ prednisone as } \\
\text { well as localized radiation }\end{array}$ & 24 & 1.12 & 0.45 & 0.42 & 0.26 \\
\hline 10 & $64, M$ & $\operatorname{IgA\kappa }$ & $2, \mathrm{AB}$ & Cyclophosphamide + dexamethasone & $6^{\text {d) }}$ & 1.15 & 0.65 & 0.46 & 0.05 \\
\hline 12 & $66, M$ & $\operatorname{lgG\kappa }$ & $2, \mathrm{RB}$ & $\begin{array}{l}\text { Localized radiation, } \\
\text { cyclophosphamide+prednisone }\end{array}$ & $6^{\text {d) }}$ & 1.17 & 0.22 & 0.65 & 0.25 \\
\hline
\end{tabular}

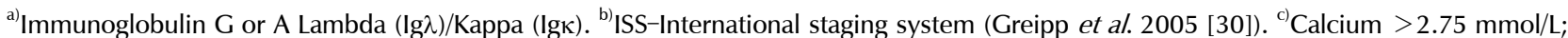
Renal: creatinine $>90 \mu \mathrm{mol} / \mathrm{L}(\mathrm{F}),>110 \mu \mathrm{mol} / \mathrm{L}(\mathrm{M})$; Anaemia: $\mathrm{HB}<12 \mathrm{~g} / \mathrm{dL}(\mathrm{F}),<13 \mathrm{~g} / \mathrm{dL}(\mathrm{M})$; Bone lesions. ${ }^{\mathrm{d})}$ Sampling period $<24 \mathrm{months}$ due to patient death. ${ }^{\mathrm{e}}$ Calculated as the $\%$ of specific cells present in the mononuclear Ficoll preparation using flow cytometry, as previously described [21]. "All MAGEC1-positive PC cells co-expressed ki-67, indicating proliferation potential [21].

Abbreviations: $F$, female; $M$, male. 
whether the simultaneous monitoring of multiple CTA transcripts would be more informative than monitoring $M A G E C 1$ alone, especially regarding clonal development and relapse prediction. Thus, the primary aim of this pilot study was to characterize CTA (MAGEA3/ABL; PRAME/ABL, and $B A G E 2 / A B L)$ expression profiles using multiplexed qRT-PCR in the $\mathrm{PB}$ of $\mathrm{MM}$ patients during chemotherapy, and to compare these data to the MAGEC1 transcript levels already established in the same patient cohort in our previous study [21]. Additionally, we aimed to investigate the clonality of the MAGEA3/PRAME/MAGEC1-expressing cell populations using flow cytometry to provide evidence of a cascade-like expression pattern occurring in stem cells during the course of the disease.

\section{MATERIALS AND METHODS}

\section{MM patient samples}

Patient samples were collected and RNA was processed as part of a previous CTA study [21]. Ethics approval for the analysis of these samples was obtained from the Human Ethics Research Committee at the University of Cape Town, Faculty of Health Sciences (HREC REF: 194/2012). Briefly, PB samples (10 mL EDTA) were collected every three months from diagnosis for up to two years during treatment with basic chemotherapy regimens (Table 1). The PB mononuclear cells (PBMCs) were isolated using density centrifugation with
Ficoll Histopaque (Sigma-Aldrich, St. Louis, MO, USA) and analyzed via flow cytometry to determine the MAGEC1positive populations as previously described [21]. The RNA was extracted using the QIAamp RNA blood mini kit (Qiagen, Hilden, Germany), treated with the TURBO DNase-free Kit (Ambion, Waltham, MA, USA) to remove residual DNA, and checked for purity and integrity (quality: 260/280 nm ratios >1.8; RNA integrity number (RIN) values: 7-10 [22], MIQE guideline compliant [23]) as per a previous study [15]. The stored RNA from 10 patients was used in the present study. Serum $M$ protein and $S \beta_{2} M$ levels were measured by the National Health Laboratory Service (NHLS)/GSH (South Africa) using accredited methodology. We compared our novel monitoring method with standardized hematological monitoring methods. Notably, the monitoring of serum free light chains was not available at our state hospitals during the study period.

\section{qRT-PCR multiplex assays}

Multiplex assays were developed and validated as per MIQE guidelines, including the assessment of amplification efficiency and specificity, and limits of detection. Reverse transcriptase (RT) reactions using $1.5 \mu \mathrm{g}$ RNA and random hexamers were performed as previously described [21]. The primers for coamplifying $M A G E A 3 / A B L, P R A M E / A B L$, and $B A G E 2 / A B L$ are listed in Table 2. PCR reactions $(25 \mu \mathrm{L})$ contained $0.8 \mathrm{mM}$ dNTPs, clear GoTaq Flexi buffer, $1.5 \mathrm{U}$ GoTaq Hotstart (Promega, Madison, WI, USA), $4 \mu \mathrm{L}$ cDNA

Table 2. Primers and probe sequences for the CTA duplex qRT-PCR assays.

MAGEA3/ABL duplex (NM_5362.3)

CTA primers

CTA probe

ABL probe

Amplicon
ABL primers

TCTTGAGCAGAGGAGTCAGCAC (F); GATCTGGTGACTCGGGAGCA (R)

56-FAM/CTCCCCCAG/ZEN/GGTGACTTCAACTA/3IABkFQ

TGGAGATAACACTCTAAGCATAACTAA (F)

GATGTAGTTGCTTGGGACCCA (R)

Cy5/CCATTTTTGGTTTGGGCTTCACACCATT/IAbRGSp

176 bp (MAGEA3)+124 bp (ABL)

PRAME/ABL duplex (NM_6115.4)

CTA primers

CTA probe

ABL primers

ABL probe

Amplicon

CTA primers
CTA probe
ABL primers
ABL probe
Amplicon

${ }^{a)}$ GenBank ref sequence ID for CTA gene.
CTGTGCTTGATGGACTTGATGTG (F)

GCTGCTCTGCCTCTGTGCTC $(\mathrm{R})$

56-FAM/ACCATCTAC/ZEN/TTTTCGCTTCTTTGTCATGGG/3IABkFQ

TGGAGATAACACTCTAAGCATAACTAA (F)

GATGTAGTTGCTTGGGACCCA (R)

Cy5/CCATTTTTGGTTTGGGCTTCACACCATT/IAbRGSp

216 bp (PRAME)+124 bp (ABL)

BAGE2/ABL duplex (NM_1839676.1)

CGGCCAGAGCGGTTTTT (F)

CTCCTCCTATTGCTCCTGTTG (R)

56-FAM/CGTCTCCAT/ZEN/CACCGTGGCTGCCACAA/IABkFG

TGGAGATAACACTCTAAGCATAACTAA (F)

GATGTAGTTGCTTGGGACCCA (R)

Cy5/CCATTTTTGGTTTGGGCTTCACACCATT/IAbRGSp

150 bp (BAGE2)+124 bp (ABL) 
(20\% of RT reaction), $4.5 \mathrm{mM}$ or $2 \mathrm{mM}$ (MAGEA3) $\mathrm{MgCl}_{2}$, $0.4 \mu \mathrm{M}$ of each CTA primer/0.2 $\mu \mathrm{M}$ CTA probe and 0.2 $\mu \mathrm{M}$ of each $\mathrm{ABL}$ primer/0.1 $\mu \mathrm{M}$ ABL probe. The cycling conditions were as follows: $95^{\circ} \mathrm{C}$ for $5 \mathrm{~min}, 40$ cycles of $95^{\circ} \mathrm{C} / 30 \mathrm{~s}, 60^{\circ} \mathrm{C} / 40 \mathrm{~s}\left(57^{\circ} \mathrm{C}\right.$ for $\left.B A G E 2\right)$, and extension at $72^{\circ} \mathrm{C} / 40 \mathrm{~s}$ ( $M A G E C 1 / A B L$ amplification was performed as per a previous study) [21]. All reactions were performed on the Rotor-Gene 6000 PCR machine (Qiagen) and analyzed using the Rotor-Gene 6000 series software 1.7. Cq values were determined using appropriate thresholds depending on the probe fluorescence. Target genes were quantified relative to $A B L$ (reference gene) expression (normalized ratio) using a modified Livak method [24], as follows: Ratio (reference/target) $=2^{\mathrm{Cq}(\text { reference)-Cq(target) }}$ or $\Delta \mathrm{Cq}$. All RNA samples were analyzed in duplicate (acceptable CV, $<20 \%$ ). The assays were able to detect $>5$ copies of each CTA transcript type; positive/negative (pos/neg) Cq cutoff values were based on assay-specific controls. $\mathrm{Cq}$ values generated from logarithmic curves were scored as positive. An ABL control indicative of 10,000 ABL copies/reaction was included in each experimental run; samples with $\mathrm{ABL} C \mathrm{Cq}<\mathrm{ABL} \mathrm{Cq}$ of controls (indicating potential RNA degradation) were excluded from further analysis.

Flow cytometry analysis of PBMCs

Approximately $2 \times 10^{6}$ ethanol-fixed PBMCs from two pa- tients prepared during the previous studies $[15,21]$ were washed twice in $40 \mathrm{~mL}$ ice-cold $1 \times \mathrm{PBS} / 1 \%$ fetal calf serum (FCS), collected via centrifugation (1,500 rpm for $5 \mathrm{~min})$, and re-suspended in $500 \mu \mathrm{L}$ cold $1 \times \mathrm{PBS} / 0.5 \%$ bovine serum albumin (BSA) buffer. Approximately $100 \mu \mathrm{L}$ of this cell suspension was incubated with the following antibody combinations for $30 \mathrm{~min}$ at $4^{\circ} \mathrm{C}$ in the dark: MAGEC1Alexa488/CD34-PE/CD45-PerCP and MAGEA3-Alexa647 or PRAME-Alexa647 and MAGEA3-Alexa647/PRAME-Alexa488/ CD34-PE/CD45-PerCP; MAGEC1-Alexa488/CD138-PE/CD45PerCP and MAGEA3-Alexa647 or PRAME-Alexa647. All antibodies were used at a final concentration of $5 \mu \mathrm{g} / \mathrm{mL}$. MAGEC1 (Abcam, clone ab115351), MAGEA3 (Abcam, clone ab38496) and PRAME (Abcam, clone ab135600) antibodies were fluorescently labeled with the Mix-n-Stain Alexa 647 kit (Sigma-Aldrich). The labeled cells were washed with $1 \mathrm{~mL}$ cold $1 \times$ PBS $/ 0.5 \%$ BSA buffer at $1,500 \mathrm{rpm}$ for $5 \mathrm{~min}$; the pellet was re-suspended in $1 \mathrm{~mL}$ cold $1 \times \mathrm{PBS} / 0.5 \% \mathrm{BSA}$ buffer and stored in the dark until analysis. The cells were analyzed on a BD FACSCalibur flow cytometer (BD Biosciences, Franklin Lakes, NJ, USA) using the Cell Quest Pro software, applying parameters and gates that had been previously optimized to minimize spectral overlap and increase sensitivity $[15,21]$.

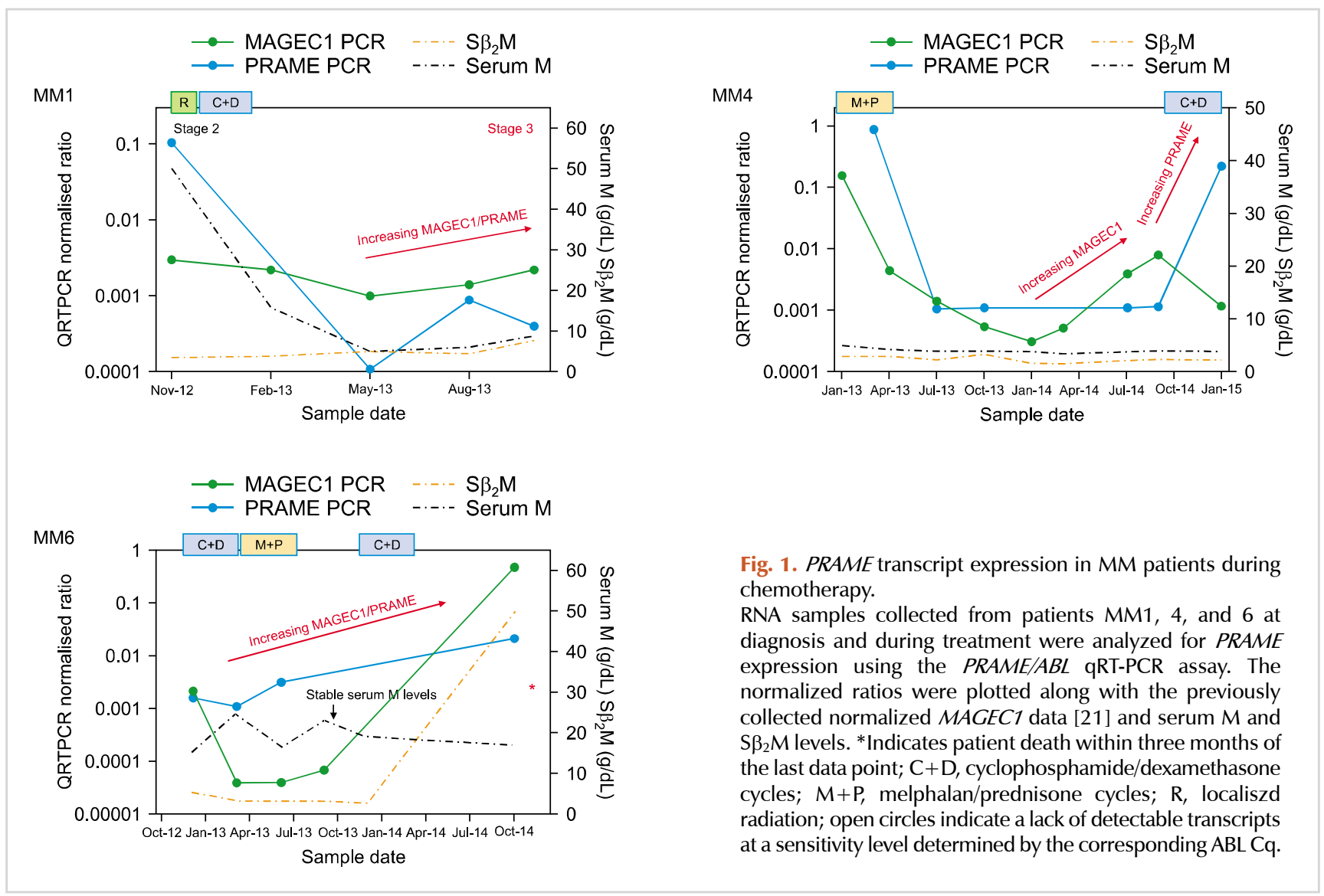




\section{RESULTS}

\section{Detection of MAGEA3, PRAME, and BAGE2 transcripts}

$P R A M E$ transcripts were detected in all the 10 patients at diagnosis, and were observed in $81 \%$ of all the RNA samples. This demonstrated the high prevalence of expression of this CTA and a possible role in early pathogenesis, in agreement with our previous findings [20]. Similarly, all patients showed MAGEC1 expression at diagnosis, and $85 \%$ of the RNA samples showed detectable transcripts [21]. In contrast, only five patients showed MAGEA3 expression at any time point (positivity rate, 20\%). However, this was not unexpected, as MAGEC1 and PRAME expression has been linked to early stage disease [17, 18, 25], and $M A G E A 3$ with more advanced disease [26, 27]. BAGE2 has been reported in advanced (stage 3) disease only [20]; in line with the above, we observed BAGE2 in three patients with clinically progressing disease.

PRAME and MAGEA3 expression levels varied widely, demonstrating positive normalized ratios over a 4-log range (0.00001-0.87 and 0.0005-4.31, respectively); these data were comparable to $M A G E C 1$ data [21]. However, BAGE2 expression was much more limited, with positive results expressed in the 2-log range (0.0008-0.0206).
Correlation of PRAME expression with clinical parameters and MAGEC1

To investigate whether monitoring $P R A M E$ transcripts could provide clinically relevant information, the expression pattern of PRAME in each patient was correlated with biochemical parameters and MAGEC1 qRT-PCR results.

$P R A M E$ expression was detected in all patient samples where serum $\mathrm{M}$ protein and/or $\mathrm{S} \beta_{2} \mathrm{M}$ levels were above the clinical threshold [28] (indicating active disease), or had stabilized or returned to baseline levels following therapy (patient achieved CR); thus this new approach has higher sensitivity for detecting residual disease than the techniques currently employed at our facilities. Significant changes in the levels of these protein markers were at least mirrored, but more importantly often preceded by changes in PRAME expression (examples shown in Figs. 1, 2). The high frequency of detection of PRAME made it an attractive target to assess circulating disease, especially where patients appeared clinically normal following therapy, but later progressed.

An example of this is shown in Fig. 1 (MM4), where the patient had stage1 disease at therapy initiation; the serum $\mathrm{M}$ levels were always within the normal range $(<8.9 \mathrm{~g} / \mathrm{dL})$ and the $\mathrm{S} \beta_{2} \mathrm{M}$ levels decreased to clinically normal levels $(<1.8 \mathrm{~g} / \mathrm{dL})$ after therapy. While PRAME levels dramatically decreased initially, it was still detectable throughout therapy (while no clinical signs of disease were recorded); a large

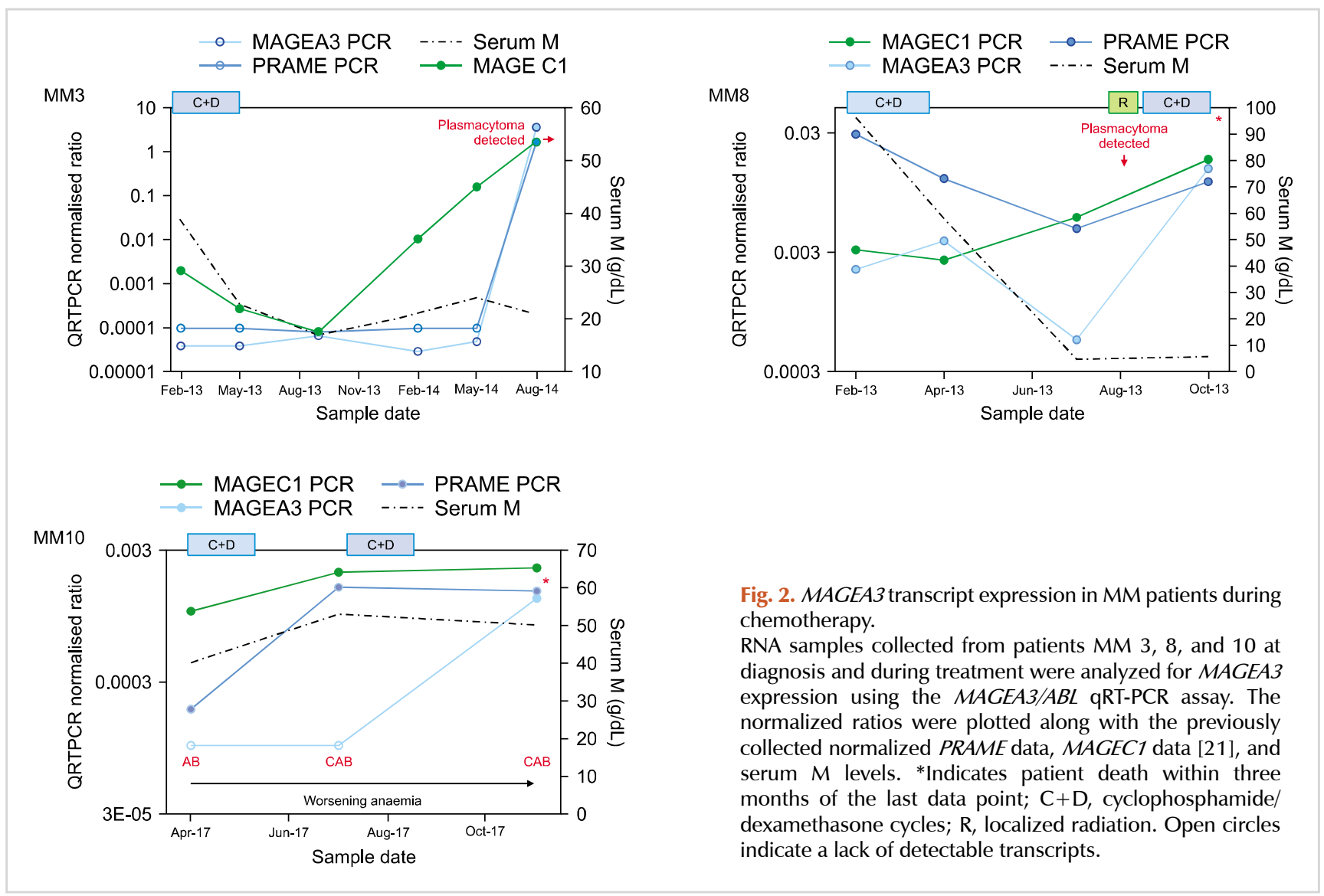


2-log increase was observed six months prior to a 10 -fold increase in paraprotein and the detection of further lytic lesions (at follow-up).

Even in cases with stable $S \beta_{2} \mathrm{M}$ and serum $\mathrm{M}$ levels following therapy for a significant period of time (Fig. 1 MM6), monitoring the patient with qRT-PCR revealed that their condition deteriorated before clinical changes were observed; a 2.6-fold increase in $P R A M E$ transcript levels was observed at least 10 months prior to the development of significant anemia (indicative of BM failure) and renal damage. The patient died soon after the last time point, demonstrating that monitoring of the $S \beta_{2} \mathrm{M}$ /serum $\mathrm{M}$ levels alone failed to predict the clinical complications early enough to avoid adverse outcomes.

$P R A M E$ and $M A G E C 1$ expression followed similar patterns in this patient cohort; $P R A M E$ levels decreased simultaneously with $M A C E 1$ in response to therapy, and increased at the same time (Fig. 1 MM1/MM6, Fig. 2 MM10) or shortly after MAGEC1 (Fig. 1 MM4, Fig. 2 MM3/MM8). Monitoring $P R A M E$ levels in conjunction with MAGEC1 levels did not offer an earlier relapse prediction compared to monitoring $M A G E C 1$ alone. However, the increases in PRAME levels were sometimes more pronounced than those in $M A G E C 1$ levels, and served to confirm $M A G E C 1$ data and disease progression. In line with the hypothesized occurrence of a CTA cascade with disease progression, $P R A M E$ expression may indicate early clonal changes that may influence malignant cell behavior.

\section{Correlation of MAGEA3 expression with clinical parameters and MAGE CI/PRAME expression}

Although the frequency of MAGEA3 detection in this MM patient cohort was only $20 \%$, any detectable expression was linked to the development of anemia $(\mathrm{Hb}$ levels $<12 / 13$ $\mathrm{g} / \mathrm{dL}$ ), with an average $\mathrm{Hb}$ level of $10.6 \mathrm{~g} / \mathrm{dL}$, as previously observed [20]. Its expression was also strongly linked to clinical progression of the disease (plasmacytoma, severe anemia, and death) (see Figs. 2, 3). Notably, in patient MM 8, MAGEA3 expression provided valuable information about disease states and imminent disease progression, which was not apparent with standard biochemical monitoring. At diagnosis, the biochemical parameters were significantly deranged, and the patient was diagnosed with stage3 (ISS) disease; elevated renal failure markers (creatinine), anemia, and bone lesions (RAB) were also observed. As per a previous report linking reduced $\mathrm{Hb}$ levels with $M A G E A 3$ expression [20], this CTA was detected at diagnosis ( $\mathrm{Hb}$ level at diagnosis, $6 \mathrm{~g} / \mathrm{dL}$ ). Following chemotherapy, the patient appeared to improve clinically (serum $\mathrm{M}$ and anemia). However, $M A G E A 3$ remained detectable and transcript levels increased
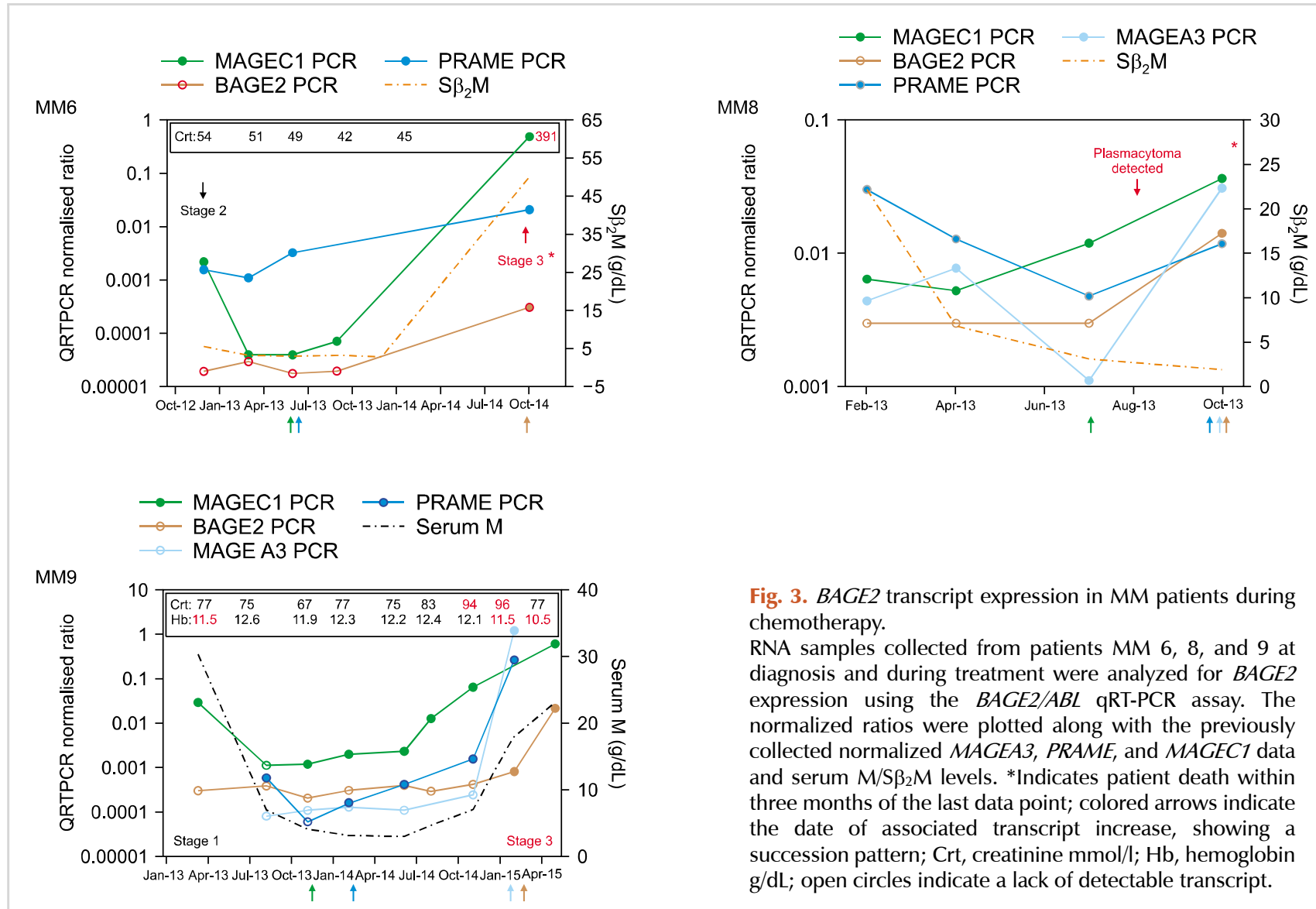

Fig. 3. BAGE2 transcript expression in MM patients during chemotherapy.

RNA samples collected from patients MM 6, 8, and 9 at diagnosis and during treatment were analyzed for $B A G E 2$ expression using the $B A G E 2 / A B L$ qRT-PCR assay. The normalized ratios were plotted along with the previously collected normalized MAGEA3, PRAME, and MACEC1 data and serum $M / S \beta_{2} M$ levels. *Indicates patient death within three months of the last data point; colored arrows indicate the date of associated transcript increase, showing a succession pattern; Crt, creatinine $\mathrm{mmol} / \mathrm{l} ; \mathrm{Hb}$, hemoglobin $\mathrm{g} / \mathrm{dL}$; open circles indicate a lack of detectable transcript. 
significantly during the last three months of the patient's disease leading up to the detection of a plasmacytoma (extramedullary disease), and the subsequently died.

The relative expression of $M A G E A 3$ was also compared to that of $M A G E C 1$ and $P R A M E$ in the relevant patients (MM 3, 4, 8, 9, and 10); while the increases in both $M A G E A 3$ and $M A G E C 1$ transcript levels correlated with imminent death or clinical relapse, the increase in MAGEC1 was detected earlier, and there were no time points at which $M A G E A 3$ was expressed in the absence of MAGEC1. $M A G E A 3$ expression increased at the same time (Fig. $2 \mathrm{MM} 3$ and MM8) or after PRAME expression increased (Fig. 2 MM10, Fig. 3 MM9). Although the sample size was small, overall MAGEA3 expression data offered no additional advantage over $M A G E C 1$ data for cell-level monitoring, but could be used to detect clonal changes that may affect therapy responses and possible spread of disease.

\section{Correlation of BAGE2 expression with MAGEC1 and clinical parameters}

Although BAGE2 expression in our patient cohort was extremely limited, its expression was clearly associated with known indicators of stage 3 disease (as per the DSS and ISS systems), namely elevated creatinine levels ( $>90$ $\mathrm{mmol} / \mathrm{L})$, severe anemia $(<10 \mathrm{~g} / \mathrm{dL})$, and/or very high $\mathrm{S} \beta_{2} \mathrm{M}$ levels $(>5.5 \mathrm{mg} / \mathrm{L})[29,30]$. Fig. 3 shows the changes in $B A G E 2$ expression in patients where it was detected (MM6, 8, and 9). Although detection of BAGE2 transcripts was always associated with a prior increase in MAGEC1 transcripts, its detection was associated specifically with advanced clinical disease indicators such as elevated creatinine (indicating organ damage), rather than just elevated PC levels (increased paraprotein). This could possibly indicate advanced clonal changes to the malignant cells in a cascade-like manner, which could potentially affect the proliferation rate, resistance to apoptosis, and thus overall drug responsiveness; these features can guide treatment for the predicted relapse. The CTA expression patterns in patient MM9 (Fig. 3) were in line with this cascade theory, and additional CTAs (MAGEC1-PRAME-MAGEA3-BAGE2) were expressed within the PBMC component as anemia, renal damage, and $\mathrm{M}$ protein levels increased.

\section{CTA co-expression in CD34+ cells}

The co-expression of multiple CTAs in this patient group indicated a cascade-like pattern as disease progressed, as previously inferred [17-20]. However, as qRT-PCR was performed on enriched PBMCs and not isolated cell populations, it was not possible to establish whether cells were undergoing sequential clonal changes, or whether different clones, or even different cell types, expressed single CTAs. Using limited residual ethanol-fixed PBMCs from two patients from the initial studies (MM3 and MM4) [15, 21] where MAGEC1-expressing cells have been extensively characterized, we additionally analyzed the expression of PRAME and MAGEA3 antigens in the matched samples (MM3 and MM4); co-expression of MAGEC1/PRAME/MAGEA3 mRNA was observed in these samples. While this study offers only preliminary results due to the limited sample pool and cell numbers, the results indicated that PRAME and MAGEA3 were only associated with the populations previously shown to express MAGEC1, namely a pro-B/pre-B progenitor population (CD34/CD19) and a PC sub-fraction (CD138+) (Table 1) [15]. We detected the co-expression of PRAME/MAGEC1 and MAGEC1/MAGEA3 in the CD34+ population and PRAME/MAGEC1 in the PC fraction (previously shown to be a proliferating population) [15]. No cells were found to express PRAME or MAGEA3 only; only small numbers of MAGEA3-positive stem cells were detected, and they were all found to be PRAME-positive, implying sequential antigen expression as opposed to activation in separate clones, although larger cell populations are required to confirm these findings.

\section{DISCUSSION}

The ability to predict and prevent relapse in MM is hindered by insufficient knowledge about the role of circulating malignant stem cells in extramedullary disease and disease progression, and clonal evolution leading to treatment resistance. Despite recent advancements, MRD monitoring techniques have limitations; affordable, non-invasive novel molecular tools that can monitor circulating disease, and can be easily applied to all patients in a standard clinic/hospital environment are needed. BM sampling for monitoring residual disease is limited in the following ways: residual disease is non-uniformly distributed in the BM, and thus, MRD can easily be missed based on the sampling site; circulating malignant cells that can lead to extramedullary disease cannot be detected. The CTAs MAGEC1, PRAME, MAGEA3, and $B A G E 2$ are commonly expressed in MM and their expression has been correlated with different disease stages. We aimed to evaluate the potential value of each of these CTAs in monitoring circulating malignant cells, and possibly predict relapse or clonal evolution of the disease at an earlier time than standard biochemical monitoring. Serum FLC analysis was not performed on this particular patient cohort, as it has only recently been introduced in the state. The availability of NGS and ASO-PCR is limited in South African, which further stresses the need for an alternative methodology that is more widely available. The approach we use here is novel, though controversial, as the PC mass was not assessed; instead, we assessed the small population of potential malignant cell progenitors circulating in the $\mathrm{PB}$ (1-2\%); we thus reveal a potential alternate monitoring target.

We included patients who were not eligible for ASCT and were treated with limited chemotherapy regimens. A significant proportion of South African patients with MM in the state health sector did not undergo ASCT because of advanced age and disease at presentation ( $>65 \mathrm{yr}$ and stage 3 disease), and comorbidities (heart disease, diabetes, and liver damage) which limit the use of myeloablative in- 
duction therapies. While most patients achieved a CR during treatment, all relapsed with extramedullary disease, and additional therapy was administered. Due to lack of novel agents for MM treatment in South African state hospitals, patients were treated primarily with cycles of cyclophosphamide and dexamethasone. Relapse in these cases was thus not unexpected. In this scenario, we aimed to assess whether qRT-PCR analysis of CTA expression could help in predicting relapse at an earlier time, allowing earlier intervention. Our previous study [21] showed that MAGEC1 could be effectively used as a monitoring tool in ASCT and chemotherapy-only patients, but as CTA expression appears to occur in a cascade-like manner with advancing disease [17-20], we wanted to investigate whether there was any clinical advantage to monitoring the expression of multiple CTAs simultaneously.

PRAME/MAGEC1 co-analysis showed promising results. We observed that all 10 patients expressed PRAME at some point during their disease, in agreement with numerous previous studies [20, 25]. qRT-PCR data showed that PRAME was always co-expressed with $M A G E C 1$, and its expression levels correlated with changes in both biochemical parameters and MAGEC1 expression. Overall, we found that $M A G E C 1 / P R A M E$ co-analysis provided a comprehensive picture of the circulating MM progenitor cells; fluctuations in the expression of these CTAs reflected clinical outcomes and frequently pre-empted changes in biochemical parameters. The expression levels increased prior to the detection of extramedullary disease (plasmacytomas) and even death, despite maintenance of CR. Preliminary flow cytometry analysis showed that PRAME is expressed in a sub-clone of MAGEC1 cells. However, its expression occurs in too many patient samples and potentially too early on in the disease to be a real indicator of significant clonal changes.

Detecting potential disease-related changes earlier than at the symptomatic level may be important in predicting response to salvage therapy at the time of relapse. Therefore, we investigated the expression of MAGEA3 and BAGE2; these CTAs are potentially expressed further down the hypothesized CTA cascade [20], and have been implicated in more advanced disease stages. MAGEA3 has been associated specifically with severe anemia [20] and stage $2 / 3$ disease. The analysis of our patient group reflected this expression pattern, and the transcript expression was detected following (or concurrently with) increases in PRAME and MAGEC1. Preliminary flow cytometry data also suggested the presence of a subpopulation of PRAME/MAGEC1+ cells expressing this third CTA, although sequential analysis of patient samples was not possible. MAGEA3 expression patterns appear to indicate clonal changes in the circulating malignant cell population (70-97\% CD34/CD19) which may be linked to relapse mechanisms and potentially affect responses to therapy. BAGE2, which has previously been linked to stage 3 disease only, was detected in three patients with clinical symptoms of disease progression, and therefore can also be potentially predictive of clonal evolution at a level further along than that of cells expressing MAGEA3. The study population was too small to compare between the CTA markers, and this needs to be studied further.

Clonal evolution of the "Myeloma cells" in MM is thought to be responsible for relapsed and treatment-refractory disease [16, 31, 32]. CTAs may be involved in this clonal evolution, leading to the development of sub-clones with higher proliferative capacity, more resistance to apoptotic signals, and refractory to inflammatory control. Although the function of many CTAs is unclear at present, members of the MAGE family are known to be involved in stimulating cell cycle progression (via cyclin activity), reduction of tumor suppression activity (P53 activity), and p21 activity via increased ubiquitination [7, 33-35]. Many CTAs are reported to be involved in overcoming cell cycle blocks via different pathways [7]. Based on our results, we may hypothesize that these clones may be highly responsive to proliferation

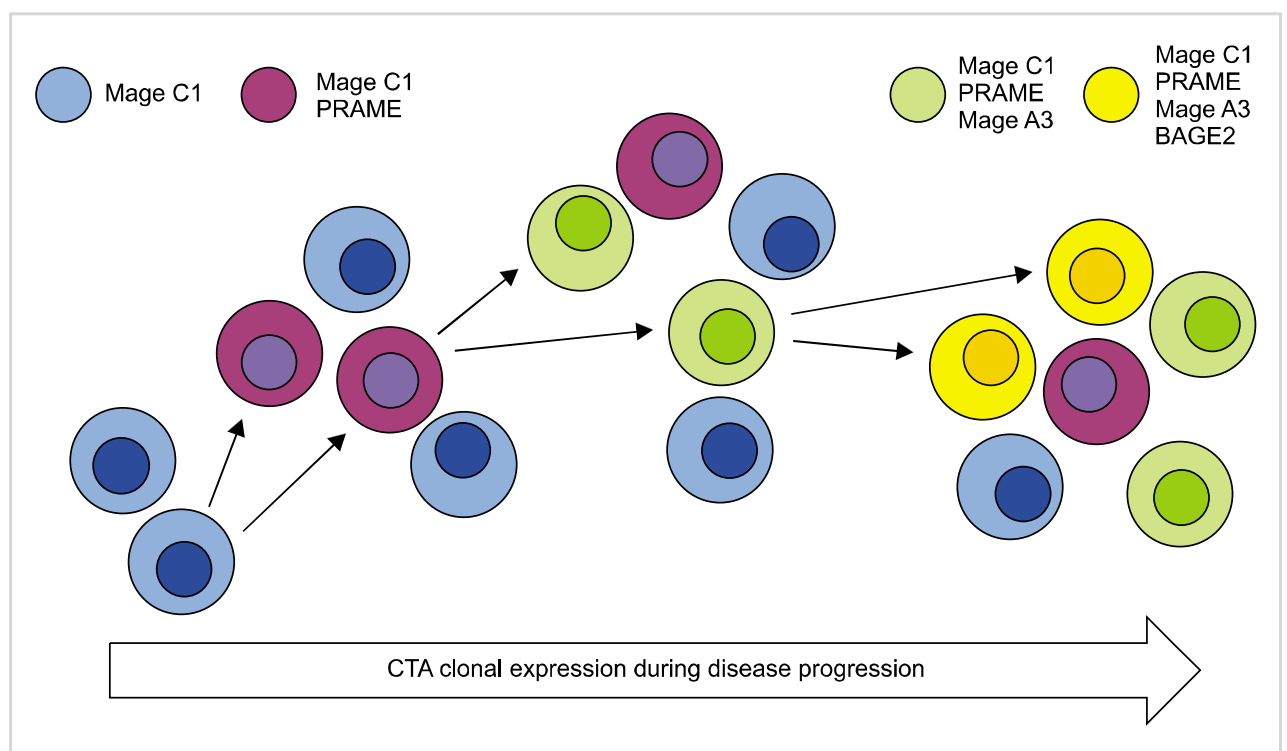

Fig. 4. Schematic of the proposed evolution of MM clones based on the expression of a CTA cascade. 
stimuli (or may proliferate without stimuli). New pathways may be activated/inhibited by the co-expression of multiple CTAs, which may influence the effectiveness of salvage therapy, depending on the drug class (i.e. proteasome inhibitors). As the cumulative effects of the CTA cascade become clear in the future, the CTA expression profiles of patients at relapse may help to guide drug selection.

Overall, this small study has provided evidence that a coordinated expansion of stem cell clones progressively expressing more CTAs may occur during disease progression in MM (Fig. 4). We also demonstrated that a multiplexed CTA qRT-PCR assay combining PRAME/MAGEC1 and $M A G E A 3$ or $B A G E 2$ can be successfully used to monitor disease with greater sensitivity than is afforded by current biochemical methods. This method provides a clearer assessment of circulating disease based on progenitor cells as opposed to considering the PC component only. However, further studies are required to determine how this novel approach compares to current MRD methodologies for predicting relapse, confirm clonal evolution through sequential patient sample monitoring (flow cytometry), and ensure that the newer biological agents such as proteasome inhibitors do not delink CTA expression from malignant cells.

CTA biomarkers linked to advanced disease may allow for the monitoring of the clonal evolution of the disease (not possible with current MRD methods), which signals the development of more aggressive disease and may in future guide treatment choices as the effect of these proteins on cellular processes is established.

\section{Authors' Disclosures of Potential Conflicts of Interest}

No potential conflicts of interest relevant to this article were reported.

\section{REFERENCES}

1. Paiva B, van Dongen JJ, Orfao A. New criteria for response assessment: role of minimal residual disease in multiple myeloma. Blood 2015;125:3059-68.

2. Nooka AK, Kastritis E, Dimopoulos MA, Lonial S. Treatment options for relapsed and refractory multiple myeloma. Blood 2015;125:3085-99.

3. Rajkumar SV, Dimopoulos MA, Palumbo A, et al. International Myeloma Working Group updated criteria for the diagnosis of multiple myeloma. Lancet Oncol 2014;15:e538-48.

4. Kumar S, Paiva B, Anderson KC, et al. International Myeloma Working Group consensus criteria for response and minimal residual disease assessment in multiple myeloma. Lancet Oncol 2016;17:e328-46

5. Keren DF, Alexanian R, Goeken JA, Gorevic PD, Kyle RA, Tomar RH. Guidelines for clinical and laboratory evaluation patients with monoclonal gammopathies. Arch Pathol Lab Med 1999; 123:106-7.

6. Davies FE, Rawstron AC, Owen RG, Morgan GJ. Minimal residual disease monitoring in multiple myeloma. Best Pract Res Clin Haematol 2002;15:197-222.

7. Shires K, Van Wyk T. The role of cancer/testis antigens in multiple myeloma pathogenesis and their application in disease monitoring and therapy. Crit Rev Oncol Hematol 2018;132:17-26.

8. Cavo M, Terragna C, Martinelli G, et al. Molecular monitoring of minimal residual disease in patients in long-term complete remission after allogeneic stem cell transplantation for multiple myeloma. Blood 2000;96:355-7.

9. Gorgun G. Predicting minimal residual disease in multiple myeloma: allelic-specific oligonucleotide real-time quantitative PCR or multi parametric flow cytometry. J Genet Syndr Gene Ther 2012;3:e105.

10. Rawstron AC, Orfao A, Beksac M, et al. Report of the European Myeloma Network on multiparametric flow cytometry in multiple myeloma and related disorders. Haematologica 2008;93: 431-8.

11. Rawstron AC, Gregory WM, de Tute RM, et al. Minimal residual disease in myeloma by flow cytometry: independent prediction of survival benefit per log reduction. Blood 2015;125:1932-5.

12. Matsui W, Huff CA, Wang Q et al. Characterization of clonogenic multiple myeloma cells. Blood 2004;103:2332-6.

13. Rasmussen T, Lodahl M, Hancke S, Johnsen HE. In multiple myeloma clonotypic CD38- /CD19+ / CD27+ memory B cells recirculate through bone marrow, peripheral blood and lymph nodes. Leuk Lymphoma 2004;45:1413-7.

14. Conway EJ, Wen J, Feng Y, et al. Phenotyping studies of clonotypic B lymphocytes from patients with multiple myeloma by flow cytometry. Arch Pathol Lab Med 2009;133:1594-9.

15. Wienand K, Shires K. The use of MAGE C1 and flow cytometry to determine the malignant cell type in multiple myeloma. PLoS One 2015;10:e0120734.

16. Johnsen HE, Bøgsted M, Schmitz A, et al. The myeloma stem cell concept, revisited: from phenomenology to operational terms. Haematologica 2016;101:1451-9.

17. Jungbluth AA, Ely S, DiLiberto $M$, et al. The cancer-testis antigens CT7 (MAGE-C1) and MAGE-A3/6 are commonly expressed in multiple myeloma and correlate with plasma-cell proliferation. Blood 2005;106:167-74.

18. Andrade VC, Vettore AL, Felix RS, et al. Prognostic impact of cancer/testis antigen expression in advanced stage multiple myeloma patients. Cancer Immun 2008;8:2.

19. Atanackovic D, Hildebrandt Y, Jadczak A, et al. Cancer-testis antigens MAGE-C1/CT7 and MAGE-A3 promote the survival of multiple myeloma cells. Haematologica 2010;95:785-93.

20. Shires K, Teuchert A, Wienand K, Shankland I, Novitzky N. Cancer/testis antigen expression panel incorporating MAGEC1 and BAGE2 predicts multiple myeloma disease stage and severity. J Hematol Thrombo Dis 2016;4:1000240.

21. Shires K, Wienand K. Cancer testis antigen MAGE C1 can be used to monitor levels of circulating malignant stem cells in the peripheral blood of multiple myeloma patients. J Cancer Res Clin Oncol 2016;142:2383-96.

22. Derveaux S, Vandesompele J, Hellemans J. How to do successful gene expression analysis using real-time PCR. Methods 2010;50:227-30.

23. Bustin SA, Benes V, Garson JA, et al. The MIQE guidelines: 
minimum information for publication of quantitative real-time PCR experiments. Clin Chem 2009;55:611-22.

24. Livak KJ, Schmittgen TD. Analysis of relative gene expression data using real-time quantitative PCR and the 2(-delta delta $\mathrm{C}(\mathrm{T})$ ) method. Methods 2001;25:402-8.

25. Pellat-Deceunynck $C$, Mellerin MP, Labarrière $N$, et al. The cancer germ-line genes MAGE-1, MAGE-3 and PRAME are commonly expressed by human myeloma cells. Eur J Immunol 2000;30:803-9.

26. Cho HJ, Caballero OL, Gnjatic S, et al. Physical interaction of two cancer-testis antigens, MAGE-C1 (CT7) and NY-ESO-1 (CT6). Cancer Immun 2006;6:12.

27. Atanackovic D, Arfsten J, Cao Y, et al. Cancer-testis antigens are commonly expressed in multiple myeloma and induce systemic immunity following allogeneic stem cell transplantation. Blood 2007;109:1103-12.

28. Swerdlow SH, Campo E, Harris NL, et al, eds. WHO classification of tumours of haematopoietic and lymphoid tissues. 4th ed. Lyon, France: IARC Press, 2008:200-13.
29. Durie BG, Salmon SE. A clinical staging system for multiple myeloma. Correlation of measured myeloma cell mass with presenting clinical features, response to treatment, and survival. Cancer 1975;36:842-54.

30. Greipp PR, San Miguel J, Durie BG, et al. International staging system for multiple myeloma. J Clin Oncol 2005;23:3412-20.

31. Morgan GJ, Walker BA, Davies FE. The genetic architecture of multiple myeloma. Nat Rev Cancer 2012;12:335-48.

32. Röllig C, Knop S, Bornhäuser M. Multiple myeloma. Lancet 2015;385:2197-208.

33. Doyle JM, Gao J, Wang J, Yang M, Potts PR. MAGE-RING protein complexes comprise a family of E3 ubiquitin ligases. Mol Cell 2010;39:963-74.

34. Richburg JH, Myers JL, Bratton SB. The role of E3 ligases in the ubiquitin-dependent regulation of spermatogenesis. Semin Cell Dev Biol 2014;30:27-35.

35. Hao J, Song X, Wang J, et al. Cancer-testis antigen MAGE-C2 binds Rbx1 and inhibits ubiquitin ligase-mediated turnover of cyclin $\mathrm{E}$. Oncotarget 2015;6:42028-39. 